DOI: https://doi.org/10.11144/Javeriana.upsy19.pirp

\title{
PANAS Internacional Revisado: Propiedades psicométricas en una muestra internacional latina*
}

\section{International PANAS Reviewed: Psychometric Properties in an International Latin Sample}

José Ignacio Ruiz-PÉrez ${ }^{a}$

Universidad Nacional de Colombia, Colombia

ORCID: https://orcid.org/0000-0002-8996-9736

VAnessa Melo-GonZÁlez

Universidad Nacional de Colombia, Colombia ORCID: https://orcid.org/0000-0002-3633-2897

Sergio Nicolás Velandia-Amaya

Universidad Nacional de Colombia, Colombia

ORCID: https://orcid.org/0000-0002-1414-0480

Luz Stella Rodríguez-Mesa

Asociación Colombiana de Criminología, Colombia ORCID: https://orcid.org/0000-0003-1933-3625

César Alfonso Velázquez Monroy Asociación Colombiana de Criminología, Colombia ORCID: https://orcid.org/0000-0003-0160-0211

a Autor de correspondencia. Correo electrónico: jiruizp@unal.edu.co

Para citar este artículo: Ruiz-Pérez, J. I., Melo-González, V., Velandia-Amaya, S. N., Rodríguez-Mesa, L. E., \& Velázquez Monroy, C. A. (2020). PANAS Internacional Revisado: Propiedades psicométricas en una muestra internacional latina. Universitas Psychologica, 19, 1-11. https://doi.org/10.11144/Javeri ana.upsy19.pirp
Recibido: 31 Julio 2020 | Aceptado: 26 Octubre 2021

\section{RESUMEN}

El objetivo de este trabajo fue analizar las propiedades psicométricas de una versión corta revisada del PANAS (Thompson, 2007), en el marco de una investigación más amplia sobre el impacto psicosocial de la pandemia por COVID-19. Se contó con una muestra internacional, especialmente de sujetos de: 1) Colombia (72\%), 2) Brasil (9.5\%), 3) otros países latinoamericanos (12.1\%) y 4) de otros países, europeos, EE. UU. y Canadá (6.31\%). Como resultados, el IPANAS-R tuvo unos coeficientes alfa de 0.82 y omega de 0.83 , sin diferencias significativas entre submuestras de acuerdo al coeficiente de Feld. Un análisis paralelo y otro factorial exploratorio muestran una solución satisfactoria de dos dimensiones, con coeficientes: RSMEA: 0.079, RMSR: 0.042, CFI: 0.949 y GFI: 0.989, aunque con diferencias de ajuste entre las submuestras. A nivel de validez convergente, un mayor apoyo social informal (familia, amistades), un mayor afrontamiento activo y una percepción del paso tiempo más positiva, rápida y esperanzadora se asociaron con un mejor balance de afectos.

\section{Palabras clave}

afectividad positiva; afectividad negativa; apoyo social; afrontamiento; COVID-19.

\begin{abstract}
The aim of this paper was to analyze psychometric properties of a review of the PANAS short form (Thompson, 2007), within the framework of the psychosocial impact of Covid-19 pandemic. Sample was international and non-randomized, withe people from 1) Colombia (72\%), 2) Brasil $(9.5 \%), 3)$ other Latinoamerican countries $(12.1 \%)$ and USA, Canada, and European countries (6.31\%). Results showed high alfa (0.82) and omega (0.82) internal reliability coefficients, without differences between groups, according Feld's test. Both parallel and exploratory factor analysis
\end{abstract}


show a bi-dimensional factorial structure, with RSMEA: 0.079, RMSR: 0.042; CFI: 0.949 and GFI: 0.989, but with important differences between simples in adjustment goodness indicators. Regarding convergent validity, high informal social support perception (relatives, friends), a high active coping and a course of time more positive, faster and hopeful were associated with a better affect balance.

Keywords

positive affect; negative affect; social support; coping; Covid-19.

El bienestar subjetivo (BS) ha sido conceptualizado como los juicios que realiza el individuo sobre su propia vida, abarcando la relación con su contexto y sus características individuales. Este se encuentra compuesto por un componente cognitivo o de satisfacción vital y un componente afectivo, o balance afectivo, los cuales intervienen en el proceso de interpretación de las condiciones vitales y su comparación con los estándares autoimpuestos por el individuo, es decir, sus expectativas en la vida. El componente afectivo se relaciona con la experimentación emocional, ya sea esta negativa o positiva (Izquierdo et al., 2008).

El afecto es una categoría global que comprende tanto las emociones como los estados anímicos (Flores-Kanter \& Medrano, 2018). En este sentido, la American Psychiatric Association (2000 citada en Vicent et al., 2016) señala que "el afecto se define como un patrón de comportamientos observables que es la expresión de sentimientos y emociones experimentados subjetivamente" (p. 334), mientras que las emociones, constituyen vivencias que ya han sido modeladas socialmente dentro de un contexto, por lo que refiere a un objeto y reacciones fisiológicas específicas (Moral de la Rubia, 2011).

Fue Bradburn (1969 citado en Otsuka et al., 2019) quien realizó los estudios pioneros del afecto, definiendo tanto el afecto positivo y negativo como sus dimensiones unipolares, de carácter dimensional no categorial e independientes entre sí. De esta manera, los afectos positivos son definidos como todos los estados afectivos con valencia positiva, placenteros, es decir la medida en que una persona se siente entusiasta, activa y alerta (Moral de la Rubia, 2011; Vicent et al., 2016) como alegría, entusiasmo, enamoramiento, etc., y los negativos, como los estados afectivos de valencia negativa tales como tristeza, ira, miedo, ansiedad, etc. (Otsuka et al., 2019).

Posteriormente, Watson y Tellegen (1985 citados en Otsuka et al., 2019), realizaron un metaanálisis enfocado en estudios en los cuales se utilizaba el autorrelato como método de recolección de información, orientado a los afectos. Utilizando análisis factoriales exploratorios, concluyeron que consistentemente aparecen dos factores: el afecto positivo y el afecto negativo, los cuales configuran finalmente la estructura básica del afecto (Flores-Kanter \& Medrano, 2018), con dos dimensiones independientes, lo que corroboraba el modelo bifactorial de la afectividad (González \& Valdés, 2015; Otsuka et al., 2019).

El Positive and Negative Affect Schedule (PANAS) fue creado a finales de los 80 por Watson et al., ( 1988), teniendo en cuenta que las mediciones de la época sobre este tema tenían poca confiabilidad y validez convergente o discriminante limitada (Otsuka et al., 2019). De una lista de 60 términos iniciales de afectos, se llegó a una lista de 20, de los cuales 10 describían afectos positivos y 10 negativos. Así, los autores aplicaron esta lista, junto con una escala Likert de cinco niveles y seis instrucciones temporales que iban desde hoy hasta el año pasado, con más de 3000 personas, para repetir la prueba después de ocho semanas. Realizaron pruebas de consistencia interna, validez testretest y estructura factorial. Así mismo, los autores encontraron correlaciones significativas entre puntajes altos en ansiedad y depresión con puntajes altos de afecto negativo, siendo esta relación inversa entre la depresión y el afecto positivo (Otsuka et al., 2019; Watson et al., 1988).

Se han realizado diversos procesos de adaptación o de validación del PANAS en diferentes países como Brasil (De Carvalho et al., 2013; Otsuka et al., 2019), Italia (Terraciano et al., 2003), Francia y Canadá (Gaudreau et al., 2006), España (Izquierdo et al., 2008; LópezGómez et al., 2015; Moral de la Rubia, 2011), 
Chile (Dufey \& Fernández, 2012), entre muchos otros, además de validaciones interculturales (Karim et al., 2011; Santángelo, Brandariz et al., 2016) orientadas, de igual manera, a diferentes poblaciones como adultos mayores (BlancoMolina \& Salazar-Villanea 2014), parejas (Moral de la Rubia, 2011) y adolescentes y niños (Sandin, 2003; Vicent et al., 2016). Además, se han adelantado estudios relacionados con las diferencias en el afecto de acuerdo con el género (Santágelo, González et al., 2016). De igual forma, el afecto medido a través del PANAS se ha utilizado como medida del efecto de las estrategias de afrontamiento proactivas y reactivas implementadas ante eventos estresantes (Smith et al., 2015).

Una versión reducida del instrumento (PANAS-I-SF) fue desarrollada por Thompson (2007), con el objetivo de estudiar su aplicabilidad en estudios interculturales. Esta versión cuenta con un total de 10 ítems distribuidos de forma equitativa en cada una de las dos dimensiones. De esta forma, la dimensión de Afecto Positivo (AP) se encuentra compuesta por los reactivos: attentive, determined, inspired, active y alert, mientras que la dimensión de Afecto Negativo (AN) se encuentra compuesta por los reactivos: afraid, nervous, upset, hostile y ashamed (Thompson, 2007).

Así mismo, Karim et al. (2011) llevaron a cabo una validación de esta escala en una muestra intercultural, enfatizando que el desarrollo de la escala se había llevado a cabo solamente en culturas occidentales, obteniendo resultados psicométricos satisfactorios en cuanto a la estabilidad de dos factores del instrumento. Si bien esta versión reducida se encuentra en el idioma inglés, en Latinoamérica, se han realizado propuestas de traducción y adaptación del instrumento como las de Galinha et al. (2014), quienes proponen la traducción del instrumento al idioma portugués y su adaptación en una muestra brasileña. De igual forma, FloresKanter y Medrano (2018) realizaron un análisis comparativo de la escala reducida en su idioma original (inglés) y su adaptación en portugués, para su aplicación y validación en la población argentina, dando como resultado que la versión portuguesa es más adecuada para esta población.

En este marco, el objetivo de este trabajo es analizar el comportamiento psicométrico de la versión PANAS-I-SF, en una muestra internacional, dado que en español latinoamericano ciertos términos pueden ser interpretados de forma equívoca en relación con la intención de la versión original. Por ejemplo, "Atento", como traducción de "Attentive" que hace parte del AP, significa en varios países de América Latina también 'educado/a' o 'cortés'.

\section{Método}

\section{Participantes}

Las 729 personas que respondieron al instrumento fueron agrupados, en cuanto a la nacionalidad, en cuatro subgrupos: Colombia ( $n$ $=526)$, Brasil $(n=69)$, países latinoamericanos $(n=88)$ y resto del mundo, mayormente sociedades de Europa occidental y norte de América $(n=46)$. Entre las sociedades latinoamericanas se encuentran El Salvador ( $n$ =39), Ecuador $(n=17)$, Argentina $(n=13)$, Panamá $(n=8)$, mientras que en el cuarto grupo se encuentran países como España $(n=$ 16), Canadá $(n=10)$, Estados Unidos $(n=6)$, Inglaterra-Gales $(n=5)$ e incluso un caso $(n=$ 1) desde Japón.

\section{Materiales e instrumentos}

\section{International Positive and Negative Affect Schedule (PANAS-I-12)}

La presente versión del PANAS se basa en la International Positive and Negative Affect Schedule Short-Form (I-PANAS-SF), propuesta por Karim et al. (2011). Esta versión se compone de diez ítems, cinco por cada dimensión de afectividad, y ha mostrado validez transcultural en estudiantes de Pakistán y de Francia. La adaptación del I-PANAS-SF para este proyecto consistió en revisar la validez de contenido y 
la comprensión adecuada en español de los ítems en inglés. De ello resultó una versión del PANAS de doce ítems, que arroja, como las versiones anteriores, tres puntajes: afectividad positiva, afectividad negativa y balance de afecto. Cada ítem presenta cuatro opciones de respuesta acerca de en qué medida la persona se ha sentido en cada aspecto últimamente, desde Nada (1) a Bastante (4). Además, se añadió "contento" a la subescala de AP y "triste" a la de AN.

\section{Fuentes de Apoyo Social}

Se contó una escala de fuentes de apoyo social elaborada para esta investigación, con 19 ítems sobre grado de apoyo estresante (2), neutro (3) o reconfortante (4) el recibido por fuentes de apoyo social como la familia, amistades, centros comunitarios, de salud, educativos o religiosos o del sector justicia y político. A partir de los datos recogidos, se construyeron tres fuentes de apoyo social: informal (familiar, amistades, vecinos, conocidos), de organizacionales sociales (salud, educación, iglesias) y sector legislativojudicial (gobiernos nacional y local, policía, ejército, etc.). La fiabilidad interna con los coeficientes alfa de Cronbach de las escalas recolectadas fue: apoyo informal: 0.68; apoyo de organizaciones sociales: 0.78 y apoyo de sector legislativo-judicial: 0.87 , mientras que los coeficientes omega resultaron en: $0.68,0.79$ y 0.89 , respectivamente.

\section{Percepción del tiempo}

A partir de la literatura científica sobre percepción del tiempo (Azzollini et al., 2004; Del Río \& Herrera, 2006), se elaboró una escala sobre percepción del paso del tiempo, con tres ítems tipo diferencial semántico de cinco puntos, para cada uno de los ítems, las personas debían indicar cómo percibían el paso del tiempo: 1) rápido-lento, 2) positivo-negativo y 3 ) esperanzadesesperanza. Cada ítem mide una dimensión específica del paso del tiempo, que se considera independiente de las demás, por lo que su uso para este trabajo se hizo de forma individual.

\section{Afrontamiento activo}

Para contrastar el estilo de afrontamiento durante el encierro con los puntajes en la PANAS-R-, se contó con el siguiente ítem: "He tomado conciencia de la situación y planeo cómo actuar". Se preguntó la frecuencia con la que la persona había recurrido a dicha estrategia, en las dos últimas semanas, desde Nunca (1) a Con mucha frecuencia (4).

\section{Procedimiento}

Una vez confeccionado el instrumento en formato de Google, se envió a diferentes grupos de WhatsApp y contactos de los autores. El formato incluía un encabezamiento en el que se explicaba el objetivo de la encuesta, asociada al nodo de Colombia de la Red Iberoamericana de Fortalecimiento de Comunidades ante Desastres Extremos (RIFOREDEX-Colombia), se solicitaba responderla voluntariamente y se informaba del uso estrictamente académico de la información. El formato incluía una sección para recoger el consentimiento informado de los participantes.

En cuanto a los análisis, en primer lugar, se realizó una descripción general de la muestra en los aspectos sociodemográficos. Seguidamente, se calcularon los índices alfa y omega, índice de homogeneidad, estadísticos básicos del IPANAS$R$ y estadístico $W$ de Feld de comparación de la fiabilidad interna (alfa de Cronbach y Omega) entre grupos, se realizó un análisis factorial exploratorio de dicha escala y se estudió su validez convergente y con indicadores de apoyo social, de afrontamiento y de percepción del tiempo.

\section{Resultados}

Comenzando por la descripción sociodemográfica de la muestra, en cuanto a la edad de sujetos, esta se compone de grupos entre 18 y 25 años ( $25.8 \%)$, entre los 26 y los 35 años (24.96\%), de 36 a 45 años (27.02 \%), de los 46 a 
los 55 años (13.85\%), entre los 56 y los 65 años (6.86 \%) y con más del 65 (1.51). Con relación a las distribuciones por género, estas son del $69.13 \%$ para mujeres y $30.86 \%$ para hombres, sin ningún sujeto marcando la opción de "otro género". En cuanto al estrato socioeconómico, el $16.46 \%$ se ubican en clase baja (estratos 1 y 2 en la taxonomía de Colombia), el $41.56 \%$ en clase media (estrato 3), el $30.59 \%$ en clase media alta (estrato 4), y el $11.38 \%$ en clase alta (estratos 5 y 6).

\section{Fiabilidad interna}

Se calcularon los índices de fiabilidad interna alfa y omega de la escala PANAS-I-R. En la Tabla 1 , se muestran los resultados, incluyendo los estadísticos básicos, el índice de homogeneidad. Una vez recodificados los ítems de estados negativos, la fiabilidad interna de toda la escala arrojó unos coeficientes $\alpha=0.776$ y $\omega=$ 0.789. Sin embargo, se puede observar que el ítem 5 ("alerta") tiene una correlación muy baja y negativa con el resto de la escala. Si se extrae este ítem, los coeficientes de fiabilidad interna suben a: $\alpha=0.807$ y $\omega=0.805$. Las medias observadas en la Tabla 1 , previas a la recodificación de los ítems negativos, muestran que en general predominan los estados positivos sobre los negativos. Las medias más altas se encuentran en "Consciente de las cosas", el ítem que sustituyó al término original "Atento". En cuanto a "Alerta", el actual contexto de crisis sanitaria, puede hacer que este sea reconocido en parte de los sujetos de la muestra más como un estado negativo que positivo, de ahí su baja correlación con el resto de la escala. Por otro lado, los coeficientes para el PANAS positivo son: $\alpha$ $=0.754$ y $\omega=0.766$ y extrayendo el ítem 5: $\alpha=0.779$ y $\omega=0.781$. En cuanto al PANAS negativo, son: $\alpha=0.812$ y $\omega=0.813$.
Tabla 1

Estadísticos básicos e índices de homogeneidad y fiabilidad de la escala PANAS-I-R

\begin{tabular}{lrrccc}
\hline \multicolumn{1}{c}{ Ítems } & Media & DE & $\begin{array}{c}\text { Índice } \\
\text { Homogeneidad }\end{array}$ & $\begin{array}{c}\boldsymbol{\alpha} \text { de } \\
\text { Cronbach }\end{array}$ & $\begin{array}{c}\boldsymbol{\omega} \text { de } \\
\text { McDonald }\end{array}$ \\
\hline 1-Activo/a & 3.03 & 0.847 & 0.528 & 0.749 & 0.763 \\
2-Nervioso/a a & 2.22 & 0.96 & 0.492 & 0.751 & 0.761 \\
3-Consciente de las cosas & 3.62 & 0.668 & 0.248 & 0.775 & 0.787 \\
4-Hóstil a & 1.78 & 0.867 & 0.397 & 0.762 & 0.771 \\
5-Alerta & 2.95 & 0.922 & -0.034 & 0.807 & 0.805 \\
6-Triste a & 2.17 & 0.995 & 0.594 & 0.739 & 0.749 \\
7-Asustado/a a & 2.13 & 0.998 & 0.427 & 0.759 & 0.767 \\
8-Decidido/a & 2.95 & 0.919 & 0.528 & 0.748 & 0.763 \\
9-Disgustado/a a & 1.94 & 0.935 & 0.484 & 0.753 & 0.762 \\
10-Inspirado/a & 2.62 & 0.957 & 0.458 & 0.755 & 0.769 \\
11-Avergonzado/a a & 1.28 & 0.633 & 0.323 & 0.769 & 0.779 \\
12-Contento/a & 2.78 & 0.888 & 0.538 & 0.747 & 0.761 \\
\hline
\end{tabular}

a Ítems revertidos: las medias son previas a la recodificación, el resto de coeficientes son posteriores a la recodificación.

Seguidamente, se exploró si había diferencias significativas entre grupos en cada una de las dimensiones o subescalas del PANAS-R-I. Para ello se calculó el coeficiente W de Feld (MerinoSoto, 2016; Merino Soto \& Lautenschlager, 2003), arrojando los resultados de la Tabla 2, en los que se excluyó el ítem 5. Según ello, la fiabilidad interna de la escala es bastante estable entre los diferentes grupos societales, sin que haya diferencian significativas entre los coeficientes de Cronbach, y sí una diferencia tendencial que apunta a una menor fiabilidad interna de la escala en la muestra de Brasil, particularmente en la dimensión positiva de la escala.

Tabla 2

Diferencias entre grupos en el alfa de Cronbach de la PANAS-I-R según el estadístico w de Feld

\begin{tabular}{lrrr}
\hline \multicolumn{1}{c}{ Submuestra } & PANAS + & PANAS & PANAS total \\
\hline Colombia & 0.788 & 0.806 & 0.806 \\
Brasil & 0.633 & 0.756 & 0.775 \\
Otros latinoamericanos & 0.762 & 0.823 & 0.763 \\
Resto del mundo & 0.823 & 0.877 & 0.836 \\
\hline$W$ de Feld $(g l=3)$ & $6.99^{*}$ & $6.65^{*}$ & $2.7 \mathrm{n} . \mathrm{s}$. \\
\hline
\end{tabular}

$* p<0.01 . g l=$ Grados de libertad.

En cuanto a la validez factorial del instrumento, se procedió a un análisis paralelo y un análisis factorial exploratorio de la escala PANS-I-R. El análisis paralelo sugiere una solución factorial de dos dimensiones, arrojando unos mejores coeficientes de ajuste si se extrae el ítem 5. La Tabla 3 presenta los resultados encontrados, con los índices de ajuste, tanto para la muestra total como por subgrupos, incluyendo 
o extrayendo el ítem 5. De otro lado, el estadístico de Bartlett para 66 grados de libertad fue 2707.8, con $p=0.00001$, mientras que índice $\mathrm{KMO}$ fue 0.832 con rangos entre 0.822 y 0.851 . En la Tabla 4, se presenta el resultado de la matriz rotada promax, extrayendo el ítem 5. Cuando se incluye, el resultado bidimensional es similar, pero se aprecia que el ítem 5 carga tanto en el factor de estados negativos (0.417) como en el factor positivo (0.488). La correlación entre ambos factores es $-0.344, p<0.05$, con rangos entre -0.439 y -0.243 . De acuerdo a las convenciones aceptadas en la comunidad científica como índices de ajustes adecuados (Stacciarini \& Pace, 2017), se considera modelo apropiado para la muestra total con extracción del ítem 5, si bien se aprecia que a nivel del coeficiente RMSEA, la bondad de ajuste del modelo es baja al separar por subgrupos.

Tabla 3

Indices de ajuste del Análisis Factorial Exploratorio con extracción de máxima verosimilitud

\begin{tabular}{lcrrrrr}
\hline Muestra total & $\boldsymbol{X}^{\mathbf{2}}$ & RMSEA & RMSR & NNFI & CFI & GFI \\
\hline Con el ítem 5 & 245.38 & 0.083 & 0.042 & 0.922 & 0.949 & 0.989 \\
Sin el ítem 5 & 184.72 & 0.079 & 0.04 & 0.936 & 0.961 & 0.991 \\
\hline Colombia & & & & & & \\
Con el ítem 5 & 244.11 & 0.095 & 0.051 & 0.898 & 0.934 & 0.984 \\
Sin el ítem 5 & 187.51 & 0.095 & 0.049 & 0.907 & 0.943 & 0.986 \\
\hline Brasil & & & & & & \\
Con el ítem 5 & 74.75 & 0.112 & 0.083 & 0.778 & 0.856 & 0.959 \\
Sin el ítem 5 & 58.61 & 0.117 & 0.084 & 0.787 & 0.868 & 0.957 \\
\hline Otros latinoamericanos & & & & & \\
Con el ítem 5 & 82.52 & 0.103 & 0.069 & 0.865 & 0.912 & 0.97 \\
Sin el ítem 5 & 63.9 & 0.101 & 0.066 & 0.882 & 0.927 & 0.978 \\
\hline Resto del mundo & & & & & & \\
Con el ítem 5 & 64.08 & 0.161 & 0.067 & 0.793 & 0.865 & 0.982 \\
Sin el ítem 5 & 47.85 & 0.122 & 0.06 & 0.891 & 0.933 & 0.988 \\
\hline
\end{tabular}

Nota. Grados de libertad de $\chi^{2}$ con el ítem $5 \mathrm{gl}=$ 43 , sin el ítem $5 \mathrm{gl}=34$. Todos las estimaciones de $X^{2}$ tienen un nivel de significancia $p<0.0001$
Tabla 4

Análisis factorial exploratorio de la PANAS-R-I. Matriz rotada promax

\begin{tabular}{lrr}
\hline \multicolumn{1}{c}{ Ítems } & Factor 1 & Factor 2 \\
\hline PANAS-01 & 0.675 & \\
PANAS-03 & 0.568 & \\
PANAS-08 & 0.75 & \\
PANAS-10 & 0.633 & \\
PANAS-12 & 0.599 & \\
PANAS-02 & & 0.739 \\
PANAS-04 & & 0.584 \\
PANAS-06 & & 0.749 \\
PANAS-07 & & 0.763 \\
PANAS-09 & & 0.605 \\
PANAS-11 & 3.795 & 0.416 \\
\hline Autovalor & 34.49 & 18.65 \\
\% de varianza explicada & & \\
\hline
\end{tabular}

Validez convergente

Para conocer las propiedades de validez convergente del PANAS-R-I en su versión de 11 ítems, se calcularon correlaciones entre los indicadores del PANAS con indicadores de apoyo social, de afrontamiento activo y de percepción del paso del tiempo (Tabla 5). Por un lado, en la muestra general, se encuentra que la afectividad positiva se relaciona inversamente con percibir el tiempo más lento, más negativo y con más desesperanza, mientras que el puntaje en afectividad negativa correlaciona directamente con estos indicadores de percepción del tiempo. $\mathrm{El}$ afrontamiento activo, entendido en este caso como tomar conciencia de la situación y planear se relaciona coherentemente con las dimensiones del PANAS, lo mismo que el indicador de apoyo social informal, es decir, a mayor afrontamiento activo y mayor apoyo percibido (reconfortante) de familia y amistades, menos afectividad negativa, más afectividad positiva y mejor balance de afectos. En cambio, es de notar que ni el apoyo percibido de organizacionales educativas, comunitarias o religiosas, ni el de instituciones judiciales y políticas, se asocia significativamente con los niveles de afectividad, salvo con un mejor balance de afectos para el apoyo de organizaciones en la muestra total. Los resultados para la muestra colombiana son 
similares a la muestra total, lo cual es lo esperable desde que más del $70 \%$ del total de sujetos son colombianos/as.

\section{Tabla 5}

Correlaciones de Pearson entre los indicadores del PANAS con percepción del tiempo, afrontamiento activo y apoyo social

\begin{tabular}{|c|c|c|c|c|c|c|c|}
\hline $\begin{array}{l}\text { Muestra } \\
\text { total }\end{array}$ & $\begin{array}{l}\text { Rápido- } \\
\text { Lento }\end{array}$ & $\begin{array}{l}\text { Positivo- } \\
\text { Negativo }\end{array}$ & $\begin{array}{l}\text { Esperanza- } \\
\text { Desespera. }\end{array}$ & $\begin{array}{l}\text { Afront. } \\
\text { Activo }\end{array}$ & $\begin{array}{l}\begin{array}{l}\text { Apoyo } \\
\text { informal }\end{array} \\
\text { in }\end{array}$ & $\begin{array}{l}\text { Apoyo } \\
\text { Organiz. }\end{array}$ & $\begin{array}{l}\text { Apoyo } \\
\text { Instituc. }\end{array}$ \\
\hline $\begin{array}{l}\text { PANAS- } \\
\text { PANAS+ } \\
\end{array}$ & $\begin{array}{l}0.148^{* * *} \\
-0.206^{* * *} \\
-0.219^{* * * *}\end{array}$ & $\begin{array}{l}0.454^{* * *} \\
-0.391^{* * *} \\
-0.525^{* * *}\end{array}$ & $\begin{array}{l}0.326^{* * *} \\
-0.372^{* * *} \\
-0.433 * * *\end{array}$ & $\begin{array}{c}-0.199^{* * *} \\
0.404^{* * *} \\
0.377^{* * *}\end{array}$ & $\begin{array}{l}-0.178^{* * *} \\
0.247^{* * *} \\
0.262 * * *\end{array}$ & $\begin{array}{l}-0.102+ \\
0.089 \\
0.19 *\end{array}$ & $\begin{array}{l}0 \\
0.04 \\
0.026\end{array}$ \\
\hline \multicolumn{8}{|l|}{ Colombia } \\
\hline PANAS- & $0.155^{* * *}$ & $0.457^{* * *}$ & $0.346^{* * *}$ & $-0.206^{* * *}$ & $-0.222 * * *$ & -0.092 & \\
\hline PANAS+ & $-0.232^{* * *}$ & $-0.412^{* * *}$ & $-0.385^{* * *}$ & $0.425^{* * *}$ & $0.2^{* * * *}$ & -0.083 & 0.02 \\
\hline Balance & $-0.241^{* * *}$ & $-0.54^{* * *}$ & $-0.0455^{* * *}$ & $0.392 * * *$ & $0.26 * * *$ & 0.11 & 0.019 \\
\hline \multicolumn{8}{|l|}{ Brasil } \\
\hline PANAS- & & $0.474^{* * *}$ & & & & & \\
\hline PANAS+ & -0.193 & $-0.428^{* * *}$ & $-0.329 * *$ & $0.423 * * *$ & 0.123 & -0.239 & -0.14 \\
\hline Balance & $-0.273^{*}$ & $-0.537 * * *$ & $-0.339 * *$ & $0.349 * * *$ & -0.086 & -0.146 & -0.137 \\
\hline \multicolumn{8}{|c|}{ Latinoamericanos } \\
\hline PANAS- & 0.084 & $0.398^{* * *}$ & $0.268^{*}$ & $-0.19+$ & -0.074 & -0.012 & 0.013 \\
\hline PANAS+ & $-0.26^{*}$ & -0.08 & -0.155 & $0.277^{* *}$ & $0.301^{*}$ & $0.328^{*}$ & 0.21 \\
\hline Balance & $-0.223^{*}$ & $-0.334^{* * *}$ & $-0.288^{* *}$ & $0.309 * *$ & $0.24+$ & 0.213 & 0.129 \\
\hline \multicolumn{8}{|c|}{ Resto del mundo } \\
\hline PANAS- & & & & & & & \\
\hline PANAS+ & -0.008 & $-0.442^{* *}$ & $-0.459 * * *$ & $0.262+$ & 0.242 & -0.044 & 0.005 \\
\hline Balance & -0.104 & $-0.456^{* * *}$ & $-0.339 *$ & 0.159 & 0.245 & 0.155 & 0.019 \\
\hline
\end{tabular}

Ahora, para el resto de subgrupos, los resultados son variables. En general, se encuentran resultados coherentes con las dimensiones del tiempo y las del PANAS, especialmente para las dimensiones de tiempo positivo-lento y esperanza-desesperanza para el PANAS positivo y negativo en la muestra brasileña, en el PANAS negativo en la muestra latinoamericana y en el PANAS positivo para el cuarto grupo. Con todo, el balance de afectos se asocia de forma similar con las dimensiones del tiempo en todos los grupos muestrales. Por su lado, el afrontamiento activo se asocia de forma similar en todas las muestras con mayores puntajes en el PANAS positivo y en balance, excepto en la muestra del resto del mundo, con una asociación solo tendencial con el PANAS positivo. En cuanto a los indicadores de apoyo social, solo en la muestra latinoamericana (además de la colombiana) presenta una correlación positiva con el PANAS positivo, lo mismo que el apoyo percibido por las organizaciones.

\section{Discusión y conclusiones}

El propósito de este trabajo fue contar con una escala breve para la evaluación de la afectividad que pueda emplearse en estudios sobre el impacto psicosocial de la pandemia por COVID-19. Para ello, se recurrió a investigar las propiedades psicométricas del PANAS-I-SF de Thompson (2007), que han sido satisfactorias en diferentes idiomas y culturas (Karim et al., 2011; Otsuka et al., 2019). A partir de la revisión lingüística y de contenido de la escala y de añadirle nuevos ítems, se obtuvo una lista de 12 ítems, de la cual, a partir de los análisis realizados, se extrae una versión final de 11 ítems. Esta versión se aplicó a una muestra compuesta en su mayoría por participantes de Colombia, Brasil y de otras latitudes, mostrando una fiabilidad interna satisfactorias, tanto a nivel global como por submuestras, para la escala total y por cada dimensión -PANAS positivo y PANAS negativo-. Aunque los indicadores de fiabilidad interna fueron más bajos para la muestra brasileña, las diferencias no fueron significativas entre muestras, de acuerdo al estadístico de Feld (Merino-Soto, 2016). El ítem eliminado, "Alerta", hace parte de la dimensión positiva del PANAS desde su formulación original. Sin embargo, en el contexto de la crisis por COVID-19 y su impacto emocional, puede hacer que este ítem tenga una connotación negativa, que lleve a que obtenga una correlación casi cero con el resto de la escala.

En cuanto a las dimensiones subyacentes a la escala, la estructura factorial se ajusta al modelo de la doble afectividad, mostrando unos coeficientes apropiados en el modelo factorial resultante para la muestra total y la colombiana, como se expone en la Tabla 3, aunque el coeficiente $X^{2}$ es significativo tanto para la muestra general como para las submuestras y a que el resto de coeficientes no es satisfactorio para las muestras brasileña, de otros países latinoamericanos y para la del resto de países.

Por un lado, como señala Herrero (2010), el coeficiente $\chi^{2}$ es más significativo a medida que el tamaño de la muestra es mayor, por lo 
que no es un buen indicador de la bondad de ajuste del modelo para muestras grandes. De otro lado, se reconoce en los resultados que el tamaño de la muestra colombiana probablemente tiene un efecto de arrastre sobre el ajuste del modelo para la muestra total. Los resultados del ajuste del modelo para las otras submuestras podrían mejorar de contar con muestras mayores, aunque a costa de aumentar la significatividad del coeficiente $X^{2}$.

Ahora, la utilidad del modelo bidimensional del PANAS se muestra también, al menos parcialmente, con su validez convergente con medidas de percepción del tiempo y especialmente de apoyo social informal y de afrontamiento activo. Así, se han encontrado relaciones entre un mayor afrontamiento adaptativo, que incluye la planificación y conductas activas y mejores indicadores de salud mental, como por ejemplo menor sintomatología depresiva (Gloria \& SteinHardt, 2014), aunque otros estudios no han encontrado esta relación (Ito \& Matsushima, 2017).

En el presente trabajo, se halla que el afrontamiento activo se asocia con un PANAS positivo más alto y con un mejor balance de afectos para prácticamente todas las muestras, y con un más bajo PANAS negativo en la muestra general, pero debido a la correlación significativa e inversa en la muestra colombiana. Respecto al apoyo social, se presentan resultados coherentes \#mayor apoyo social informal reconfortante y menores niveles de afectividad negativa y mayores de afectividad positiva\# solo en la muestra total y en la colombiana y en la de otros países latinoamericanos para el PANAS positivo. Es posible que la crisis sanitaria por COVID-19, en la que ha habido llamamientos de organizaciones internacionales como la Organización Mundial de la Salud (2020) a comunicarse con familiares para interesarse por ellos y su salud haya afectado la percepción del apoyo social familiar y de amistades que mide el indicador de apoyo social informal empleado en este trabajo.

De otro lado, el apoyo social de organizaciones comunitarias solo se relaciona con un mayor afecto positivo en la muestra de otros países latinoamericanos, mientras que el apoyo de instituciones judiciales y políticas no se relaciona en ningún caso con los indicadores de afectividad. Esto podría ser debido a varias causas, como el impacto que la crisis por COVID-19 ha tenido en muchas instituciones privadas y públicas, planteando enormes desafíos para su funcionamiento diario y el cumplimiento de sus misiones, por ejemplo, para los centros educativos y de salud. Las instituciones políticas y judiciales son percibidas negativamente en muchos países de la región latinoamericana: en 11 países de la región, la mitad o más de la población han creído que los políticos, incluso los presidentes, estaban implicados en la corrupción en el 2018, en 17 de 19 países la aprobación del gobierno era inferior al $50 \%$ de los encuestados, y la confianza en la policía y en el poder judicial eran del $35 \%$ y del $24 \%$, respectivamente (Corporación Latinobarónetro, 2018). De otro lado, y aun a riesgo de ser reiterativos, el impacto de la crisis por COVID-19 se ha dado en estas instituciones políticas y judiciales, obligadas a reaccionar con rapidez emitiendo decretos de confinamiento y otras normas relativas a disminuir el riesgo de contagios, y a proveer a los países de recursos para la detección de contagio, y atención a las personas enfermas en contextos de carencias de instalaciones suficientes y de recursos materiales y humanos en la mayoría de ellos. Por último, las dimensiones de percepción del paso del tiempo incluidas en el presente trabajo, muestran una relación coherente con la afectividad, siendo esta más positiva (a nivel de balance de afectos) cuando el paso del tiempo se percibe más positivo, con más esperanza y, en menor medida, más rápido en su transcurso.

El presente estudio cuenta con muestras desbalanceadas por tamaño en cuanto a los países participantes, por lo que cualquier conclusión general debe ser tomada con precaución en relación con la escala resultante del proceso de análisis. Muestras mayores pueden confirmar por subgrupos regionales las propiedades psicométricas básicas de la versión del PANAS-I-R (ver Anexo) que, con todo, se ofrece en este artículo como una medida breve de la afectividad que pueda ser empleada 
en aplicaciones sobre el impacto psicosocial de la pandemia por COVID-19, tanto en estudios transversales como longitudinales sobre el bienestar subjetivo, la afectividad y las variables asociadas a lo largo del tiempo con la salud mental de la ciudadanía.

\section{Agradecimientos}

Los autores manifiestan no tener conflicto de intereses. Agradecemos a los y las colegas colaboradores/as de los diferentes países que contribuyeron a la difusión del formato de encuesta y, por tanto, a la recolección de las muestras.

\section{Referencias}

Azzollini, S., González, F., \& Gómez, M. E. (2004). La relación entre la capacidad de atención dividida y la estimación prospectiva del tiempo. Interdisciplinaria, $21(2), 195-212$. https://www.redalyc.org/ pdf/180/18021203.pdf

Blanco-Molina, M., \& Salazar-Villanea, M. (2014). Escala de Afectividad (PANAS) de Watson y Clark en Adultos Mayores. En V. Smith-Castro (Comp.), Compendio de instrumentos de medición IIP-2014(pp. 72-76). Universidad de Costa Rica.

Corporación Latinobarómetro. (2018). Informe 2018. Autor. http://www.latinobarometro.o rglatdocs/INFORME_2018_LATINOBA ROMETRO\%20(1).pdf

De Carvalho, H. W., Andreoli, S., Lara, D., Patrick, C., Quintana, M. I., Bressan, R. A, Mello M. F., Mari, J., \& Jorge, M. R. (2013). Structural validity and reliability of the Positive and Negative Affect Schedule (PANAS): Evidence from a large Brazilian community sample. Revista Brasileira de Psiquiatria, 35, 169-172. https://doi.org/10. 1590/1516-4446-2012-0957

Del Río-González, A. M., \& Herrera, A. N. (2006). Desarrollo de un instrumento para Evaluar perspectiva de tiempo futuro en adolescentes.
Avances en Medición, 4(1), 4760. https://www.humanas.unal.edu.co/lab psicometria/application/files/3616/0463/35 38/Vol_4._Desarrollo_De_Un_Instrume nto_Para_Evaluar_Perspectiva_De_Tiemp o_Futuro_En_Adolescentes.pdf

Dufey, M., \& Fernández, A. (2012). Validez y confiabilidad del Positive Affect and Negative Affect Schedule (PANAS) en estudiantes universitarios chilenos. Revista Iberoamericana de Diagnóstico y Evaluación - e Avaliação Psicológica. .(34), 157-173. https://www.redalyc.org/pdf/4596 /459645438008.pdf

Flores-Kanter, P., \& Medrano, L. (2018). Comparación de dos Versiones Reducidas de la Escala PANAS: análisis factoriales en una muestra argentina. Revista Iberoamericana de Diagnóstico y Evaluació., 49(4), 37-46. https://doi.org/10.21865/RID EP49.4.03

Galinha, C., Pereira, R., \& Esteves, F. (2014). Versão reduzida da escala portuguesa de afeto positivo e negativo - PANAS-VRP: Análise fatorial confirmatória e invariância temporal. Psicología, 28(1), 50-62. https://d oi.org/10.17575/rpsicol.v28i1.622

Gaudreau, P., Sanchez, X., \& Blondin, J. P. (2006). Positive and negative affective states in a performance related setting: Testing the factorial structure of the PANAS across two samples of FrenchCanadian participants. European Journal of Psychological Assessment, 22 (4), 240-249. ht tps://doi.org/10.1027/1015-5759.22.4.240

Gloria, C. T., \& Steinhardt, M.A. (2014). Relationships among positive emotions, coping, resilience and mental health. Stress E3 Health, 32(2), 145-156. https://doi.org/1 $0.1002 /$ smi.2589

Herrero, J. (2010). El análisis factorial confirmatorio en el estudio de la estructura y estabilidad de los instrumentos de evaluación: un ejemplo con el Cuestionario de Autoestima CA-14. Psychological Intervention, 19(3), 289-300. https://doi.org /10.5093/in2010v19n3a9 
José Ignacio Ruiz-Pérez, Vanessa Melo-González, Sergio Nicolás Velandia-Amaya, et al.

Ito, M., \& Matsushima, E. (2017). Presentation of coping strategies associated with physical and mental health during health checkups. Community Mental Health Journal, 53, 297-305. https://doi.org/10.1007/s10597-0 16-0048-9.

Izquierdo, D., Godoy, J., \& Martínez, A. (2008). La Escala de Balance Afectivo. Propiedades psicométricas de un instrumento para la medida del afecto positivo y negativo en población española. Clínica y Salud, 19(2), 157-189. http://scielo.isciii.es/pdf/clinsa/v1 9n2/v19n2a02.pdf

Karim, J., Weisz, R., \& Rehman, S. (2011). International positive and negative affect schedule short-form (I-PANAS-SF): Testing for factorial invariance across cultures. Procedia - Social And Behavioral Sciences, 15, 2016-2022. https://doi.org/10. 1016/j.sbspro.2011.04.046

López-Gómez, I., Hervás, G., \& Vázquez, C. (2015). Adaptación de la "Escala De Afecto Positivo y Negativo" (PANAS) en una muestra general española. Behavioral Psychology/Psicología Conductual, 23(3), 529-548. https://www.behavioralpsycho.co $\mathrm{m} /$ producto/adaptacion-de-las-escalas-de-a fecto-positivo-y-negativo-panas-en-una-m uestra-general-espanola/

Merino-Soto, C. (2016). Diferencias entre coeficientes alfa de Cronbach, con muestras y partes pequeñas: un programa $\mathrm{VB}$. Anales de Psicología, 32(2), 587-588. https://doi.or $\mathrm{g} / 10.6018 /$ analesps.32.2.203841

Merino-Soto, C., \& Lautenschlager, G. J. (2003). Comparación estadística de la confiabilidad alfa de Cronbach: aplicaciones en la medición educacional y psicológica. Revista de Psicología de la Universidad de Chile, 12(2), 127-136. https://doi.org/10.5354/07 19-0581.2003.17668

Moral de la Rubia, J. (2011). La escala de afecto positivo y negativo (PANAS) en parejas casadas mexicanas. CIENCIA ergosum, 18(2), 117-125. https://cienciaergosu m.uaemex.mx/article/view/7409

Organización Mundial de la Salud. (2020). Brote por enfermedad del coronavirus (COVID-19):
Orientaciones al Público. Autor. https://www .who.int/es/emergencies/diseases/novel-cor onavirus-2019/advice-for-public

Otsuka, L., Campos, D., De Castro, R., Brandão, C., \& Pires dos Santos, P. (2019). Análisis psicométrico de la PANAS en Brasil. Ciencias Psicológicas, 13(1), 45-55. https://d oi.org/10.22235/cp.v13i1.1808

Sandin, B. (2003). Escalas PANAS de Afecto Positivo y Negativo para Niños y Adolescentes (PANASN). Revista de Psicopatología y Psicología Clínica, 8(2), 173-182. https://doi.org/10.5944/rppc.vol.8 .num.2.2003.3953

Santángelo, P., Brandariz, R., \& Cuesta, J. (2016). Análisis de la validación transcultural del PANAS (Positive and Negative Affectivity Scale). Revista Argentina de Ciencias del Comportamiento, 8(1), 176-177. https://opac.elte.hu/PrimoRecord /dialnetART0001389218

Santángelo, P., González, R., Brandariz, R., \& Conde, K. (2016). Afectividad y género: un estudio empírico. Revista Argentina de Ciencias del Comportamiento, 8(1), 177-178. https://opac.elte.hu/PrimoRecord /dialnetART0001389219

Smith, M., Saklofske, D., Keefer, K., \& Tremblay, P. (2015). Coping strategies and psychological outcomes: The moderating effects of personal resiliency. The Journal of Psychology, 150(3), 318-332. https://doi.org /10.1080/00223980.2015.1036828

Stacciarini, T. S. G., \& Pace, A. E. (2017). Análisis factorial confirmatorio de la escala Appraisal of Self care Agency Scale - Revised. Revista Latino-Americana de Enfermagem, 25, e2865. https://doi.org/10.1 590/1518-8345.1378.2856

Terraciano, A., McCrae, R., \& Costa, P. (2003). Factorial and Construct Validity of the Italian Positive and Negative Affect Schedule (PANAS). European Journal of Psychological Assessment, 19(2), 131-141. ht tps://doi.org/10.1027//1015-5759.19.2.131

Thompson, E. R. (2007). Development and validation of an internationally reliable Short-Form of the Positive and Negative 
Affect Schedule (PANAS). Journal of Cross-Cultural Psychology, 38(2), 227-242. h ttps://doi.org/10.1177/0022022106297301

Vicent, M., Gonzálvez, C., Sanmartín, R., García-Fernández, J., \& Inglés, C. (2016). Perfeccionismo socialmente prescrito y afecto en la infancia. International Journal of Developmental and Educational Psychology, 1(1), 333-339. https://doi.org/10.17060/ijo daep.2016.n1.v1.183

Watson, D., Clark, L. A., \& Tellegen, A. (1988). Development and validation of brief measures of Positive and Negative Affect - The PANAS Scales. Journal of Personality and Social Psychology, 54(6), 1063-1070. https://doi.org/10.1037/0022-3 514.54.6.1063

\section{Notas}

* Artículo de investigación. 